\title{
Long-term strength and creep resistance of a polycrystalline Re-containing nickel-based superalloy
}

\author{
Sh. Kh. Mukhtarov ${ }^{\dagger}$ V. M. Imayev, R. V. Shakhov, A. A. Ganeev \\ †shamilm@imsp.ru
}

Institute for Metals Superplasticity Problems of RAS, Ufa, 450001, Russia

\begin{abstract}
The work was devoted to the study of the long-term strength and creep resistance of the recently developed heat resistant nickel-based superalloy SDZhS-15 intended for manufacturing of discs for gas turbine engines. The as-cast alloy was subjected to homogenization heat treatment, hot forging at subsolvus temperatures with intermediate annealing, solution treatment at various temperatures and aging or only to aging. A predominantly fine-grained structure was obtained in the workpieces after forging. It was revealed that solution treatment at $T>T_{s}-50$, where $T_{s}$ is the solvus temperature of the $\gamma^{\prime}$-phase, led to a decrease in the volume fraction of the primary $\gamma^{\prime}$-phase and an increase in the volume fraction of the $\gamma^{\prime}$ precipitates together with a significant growth of $\gamma$ grains (up to $d>50 \mu \mathrm{m}$ ). Solution treatment at $T=T_{s}-50$ allowed maintaining a relatively finegrained structure $\left(d_{\gamma}=10-20 \mu \mathrm{m}\right)$ and ensured the precipitation of the secondary $\gamma^{\prime}$-phase with a size of about $0.1 \mu \mathrm{m}$ upon cooling in air. Three microstructure conditions were obtained, for which long-term strength and creep resistance tests were performed in the range of temperatures $650-850^{\circ} \mathrm{C}$ and stresses $400-1200 \mathrm{MPa}$. The highest values of the long-term strength were achieved for a relatively fine-grained condition obtained after solution treatment at $T_{s}-50$ and aging. To evaluate the service life of the superalloy, the methodology based on the calculation of the Larson-Miller parameter was used. It was shown that the long-term strength (creep resistance) of the SDZhS-15 alloy in the optimal condition was higher as compared to the industrial disc nickel-based superalloys EP741NP and Udimet 720. Microstructure examination of the creep tested samples did not result in significant microstructure changes and especially in the formation of topologically close-packed phases. After creep tests, microcracks were observed along the grain/interphase boundaries, which apparently resulted from the development of boundary diffusion and sliding.
\end{abstract}

Keywords: nickel base superalloy, solid solution treatment, microstructure, long-term strength, creep resistance.

УДК: $669.245,621.7 .043$

\section{Длительная прочность и сопротивление ползучести поликристаллического Re-содержащего никелевого сплава}

\author{
Мухтаров Ш. Х. ${ }^{\dagger}$, Имаев В. М., Шахов Р. В., Ганеев А. А. \\ Институт проблем сверхпластичности металлов РАН, Уфа, 450001, Россия
}

Работа посвящена исследованию длительной прочности и сопротивления ползучести недавно разработанного жаропрочного никелевого сплава СДЖС-15, предназначенного для изготовления дисков газотурбинных двигателей. Сплав в литом состоянии был подвергнут гомогенизационной термической обработке, деформационно-

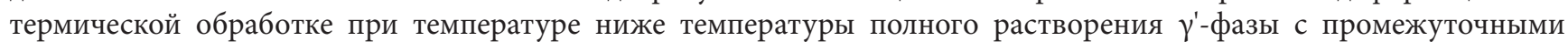
отжигами, обработке на твердый раствор при различных температурах и старению или только старению. После деформационно-термической обработки в заготовках была получена преимущественно мелкозернистая структура.

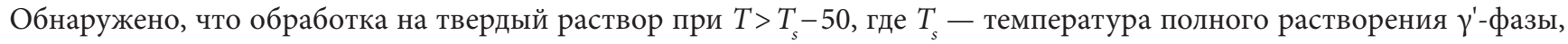
приводит к уменьшению объемной доли первичной $\gamma^{\prime}$-фазы и увеличению объемной доли дисперсной $\gamma^{\prime}$-фазы при значительном росте $\gamma$-зерен (до $d>50$ мкм). Температура обработки на твердый раствор $T=T_{s}-50$ позволяет сохранить относительно мелкозернистую структуру $\left(d_{\gamma}=10-20\right.$ мкм) и обеспечивает выделение вторичной $\gamma^{\prime}$-фазы размером около 0.1 мкм при охлаждении на воздухе. Были получены 3 состояния сплава, для которых были выполнены испытания на длительную прочность и сопротивление ползучести в диапазоне температур 650-850 $\mathrm{C}$ и напряжений 400-1200 МПа. Наибольшие значения длительной прочности были достигнуты 
для относительно мелкозернистого состояния сплава, полученного после обработки на твердый раствор при $T-50$ и старения. Для оценки срока службы сплава использовали методологию, основанную на расчете параметра ЛарсонаМиллера. Показано, что по длительной прочности (сопротивлению ползучести) сплав СДЖС-15 в оптимальном состоянии превосходит промышленные дисковые никелевые сплавы ЭП741НП и Udimet 720. Микроструктурные исследования разрушенных образцов показали, что длительная выдержка образцов под напряжением не приводит к значительным изменениям микроструктуры и, в частности, к образованию топологически плотноупакованных фаз. После испытаний на длительную прочность вдоль межзеренных/межфазных границ наблюдались микротрещины, образовавшиеся, по-видимому, в результате развития диффузии и проскальзывания по границам.

Ключевые слова: жаропрочный никелевый сплав, обработка на твердый раствор, микроструктура, длительная прочность, сопротивление ползучести.

\section{1. Введение}

Изготовление дисков для газотурбинных двигателей (ГТД) [1], как правило, предполагает деформационный передел, в процессе которого важно обеспечить не только требуемую форму заготовки, но и однородную проработку структуры с формированием рекристаллизованной мелкозернистой структуры, которая является ключом к получению регламентированных микроструктурных состояний в дисках из жаропрочных никелевых сплавов (ЖНС).

Одним из недавно разработанных дисковых ЖНС является сплав СДЖС-15. Для этого сплава ранее были разработаны условия гомогенизационной обработки и дробной деформационно-термической обработке (ДТО) в $\left(\gamma+\gamma^{\prime}\right)$-области с промежуточными отжигами, которые позволили получить поковки с преимущественно рекристаллизованной мелкозернистой структурой $[2,3]$. Сплав даже в деформированном мелкозернистом состоянии (после старения) показал повышенные механические свойства в сравнении с известными дисковыми ЖНС [2]. Тем не менее, чтобы получить сбалансированные механические свойства, в частности, более высокую длительную прочность и сопротивление ползучести, мелкозернистый сплав СДЖС-15 должен быть подвергнут обработке на твердый раствор и старению для получения высокой объемной доли дисперсных выделений $\gamma^{\prime}$-фазы [4-6]. При этом следует избегать значительного роста $\gamma$-зерен. По данным работ [7-9], сохранение мелкозернистой структуры $\left(d_{\gamma}=10-20\right.$ мкм) особенно важно для ступичной части дисков ГТД, от которой в процессе эксплуатации требуются одновременно высокая прочность, ударная вязкость, пластичность, сопротивление усталостному разрушению и термоциклическим нагрузкам $[4,5,10]$.

В настоящей работе изучалось влияние температуры обработки на твердый раствор с последующим старением на микроструктуру и длительную прочность (сопротивление ползучести) сплава СДЖС-15, подвергнутого ДТО. Длительную прочность и сопротивление ползучести сплава оценивали в различных микроструктурных состояниях.

\section{2. Материал и методики эксперимента}

Объектом исследования является поликристаллический дисковый ЖНС СДЖС-15 состава $\mathrm{Ni}-28(\mathrm{Cr}, \mathrm{Co})-$ 12.5(Al, Ti, Nb, Ta)-9(Mo, W, Re)-0.17(C, La, Y, Ce, B) (вес.\%), изготовленный с помощью вакуумного индукционного переплава. Температура полного растворения $\gamma^{\prime}$-фазы сплава составила $T_{s}=1220 \pm 5^{\circ} \mathrm{C}$ [2]. Для сплава были разработаны режимы гомогенизационного отжига, ДТО, обработки на твердый раствор и старения [2,3]. Эти режимы были использованы для получения модельных заготовок размером $\varnothing 100 \times 15$ мм и структурой, различающейся по размеру $\gamma$-зерен и объемной доле первичной $\gamma^{\prime}$-фазы.

Термическая обработка после ДТО включала в себя либо обработку на твердый раствор при 1170 и $1200^{\circ} \mathrm{C}$ с последующим охлаждением на воздухе и двухстадийным старением при температурах 860 и $750^{\circ} \mathrm{C}$ (состояния 2 и 3 , соответственно), либо только старение (состояние 1).

Микроструктурные исследования были проведены на сканирующем электронном микроскопе Mira-3 Tescan (SEM) в режиме вторичных (SE) и обратно-рассеянных электронов (BSE). Анализ дифракции обратно-рассеянных электронов (EBSD-анализ) был выполнен с шагом сканирования 0.5 мкм.

Для полученных состояний сплава были выполнены испытания на длительную прочность (сопротивление ползучести) в диапазоне температур $650-850^{\circ} \mathrm{C}$ и нагрузок 350-1200 МПа. Для этого из термически обработанных заготовок были изготовлены плоские образцы с размерами рабочей части $17 \times 3 \times 3$ мм $^{3}$. Образцы перед испытаниями механически шлифовали. Испытания проводили на испытательной машине ATS 2330. Зависимости Ларсона-Миллера (LMP) для прогнозирования продолжительности испытания образцов сплава до разрушения рассчитывали по формуле [11-13]: $\quad \mathrm{LMP}=T \times\left[\log t_{\text {раз }}+20\right]$, где $T-$ абсолютная температура испытания на длительную прочность, $t_{\text {раз }}$ - время до разрушения образца $[9,10]$. Механические испытания проводили на воздухе.

\section{3. Результаты и обсуждение}

\section{1. Микроструктура сплава после ДТО и термической обработки}

На Рис. 1 показана микроструктура центральной части заготовки сплава после ДТО и старения (состояние 1). По EBSD-карте видно, что микроструктура в основном рекристаллизованная, мелкозернистая. Средний размер рекристаллизованных $\gamma$-зерен составил $d_{\gamma}=12 \pm 1.2$ мкм. В структуре также наблюдались отдельные крупные $\gamma$-зерна размером 30-100 мкм. Доля высокоугловых 
границ составила 80\%, малоугловые границы наблюдались только в крупных $\gamma$-зернах (Рис. 1a). Первичные выделения $\gamma^{\prime}$-фазы, не растворившиеся при ДТО, имели размер 2-10 мкм, занимали около 15 об.\% и располагались в основном по границам $\gamma$-зерен (Рис. 1b). Вторичные и третичные выделения $\gamma^{\prime}$-фазы размером $d_{\gamma^{\prime}}=0.15 \pm 0.02$ мкм и $d_{\gamma^{\prime}}=0.05 \pm 0.01$ мкм, соответственно, образовались при охлаждении на воздухе от температуры деформационной обработки и при старении (Рис. 1с). Третичные выделения $\gamma^{\prime}$-фазы наблюдались по границам $\gamma$-зерен, их объемная доля не превышала $1.5 \%$. В микроструктуре присутствовали карбиды (светлая фаза) сферической и пластинчатой формы сложного состава $\left(\mathrm{Me}_{x} \mathrm{C}_{y}\right)$ размером 2-10 мкм. Их объемная доля составила около $2 \%$.
Как известно $[1,14]$ при обработке на твердый раствор может развиваться статическая рекристаллизация, которая приводит к нежелательному росту $\gamma$-зерен. В состоянии 2 объемная доля первичной $\gamma^{\prime}$-фазы составила $10-15 \%$, средний размер $\gamma$-зерен существенно не изменился по сравнению с состоянием 1 и составил $d_{v}=16 \pm 1.5$ мкм (Рис. 2a,b). Повышение температуры обработки на твердый раствор до $1200^{\circ} \mathrm{C}$ привело к росту $\gamma$-зерен за счет растворения $\gamma^{\prime}$-фазы, которая ограничивала рост $\gamma$-зерен, а также развитию статической рекристаллизации. В состоянии 3 объемная доля первичной $\gamma^{\prime}$-фазы уменьшилась до $4.5 \%$, а средний размер $\gamma$-зерен увеличился до $d_{\gamma}=60 \pm 5$ мкм (Рис. 2c). Средний размер вторичных выделений $\gamma^{\prime}$-фазы в состояниях 2 и 3 составил $d_{\gamma^{\prime}}=0.13 \pm 0.01$ и $0.12 \pm 0.01$ мкм, соответственно

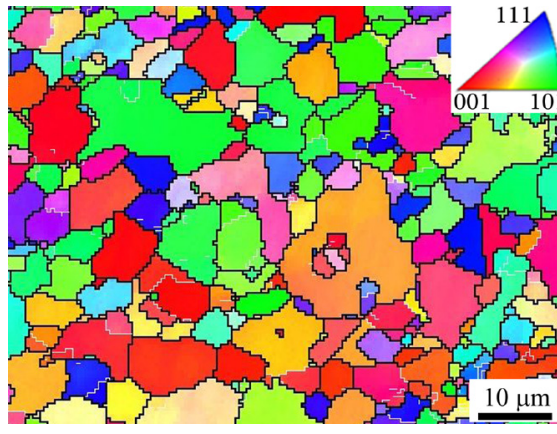

a

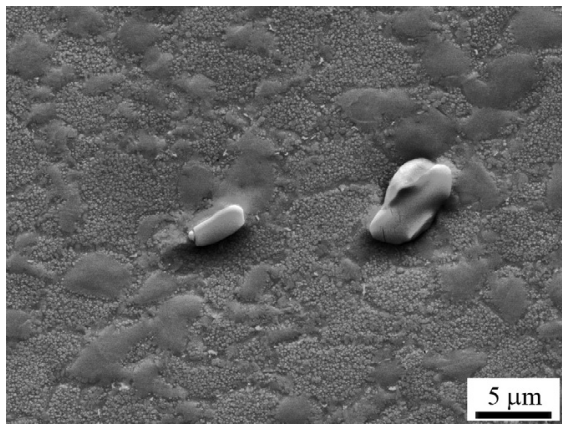

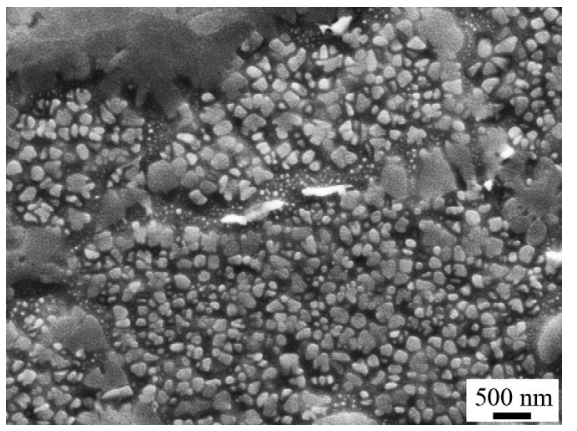

Pис. 1. (Color online) Микроструктура сплава после ДТО и старения (состояние 1): EBSD-карта в нормальном направлении (обратная полюсная фигура), ось штамповки вертикальна, высокоугловые и малоугловые межзеренные/межфазные границы обозначены черными и белыми линиями, соответственно (a); SE изображения, показывающие первичную, вторичную, третичную $\gamma^{\prime}$-фазу и белые карбидные частицы (b, c).

Fig. 1. (Color online) The microstructural images of the superalloy after hot forging and ageing (condition 1): normal-direction EBSD (inverse-pole-figure) map, the forging axis is vertical, high- and low-angle grain/interphase boundaries are indicated by black and white lines, respectively (a); SE images illustrating the primary, secondary, tertiary $\gamma^{\prime}$ phase and white carbide particles (b, c).
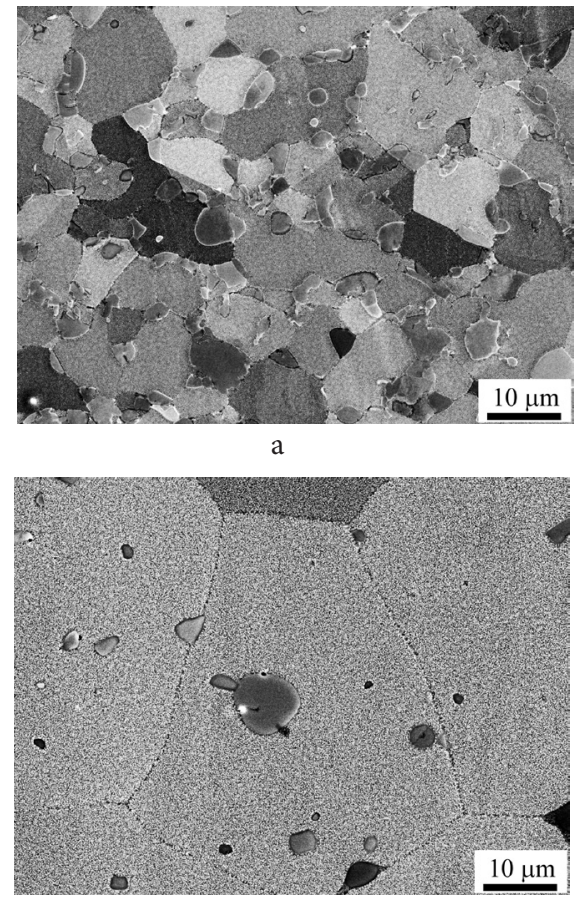

c
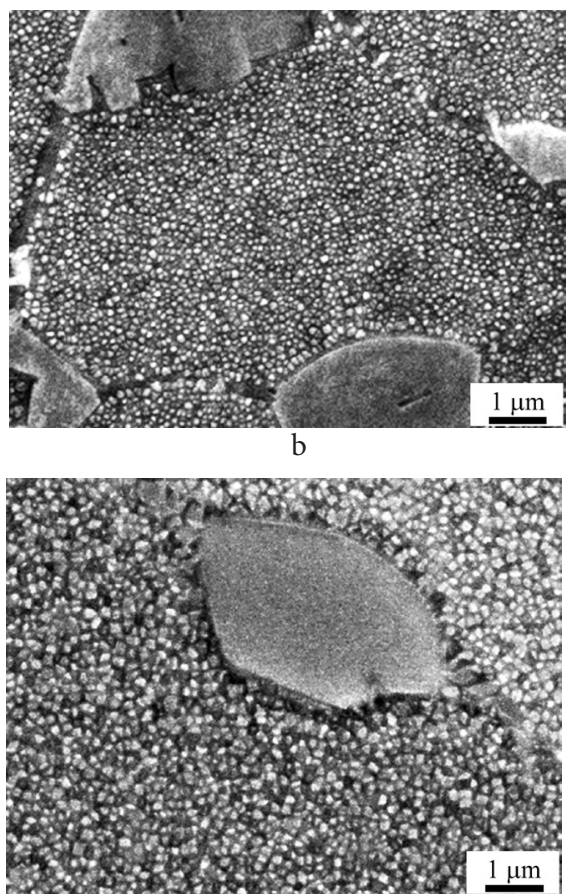

d

Рис. 2. Микроструктура (BSE) сплава СДЖС-15: состояние 2 (a, b), состояние 3 (c, d).

Fig. 2. The microstructural images of the superalloy SDZhS-15: condition 2 (a, b), condition 3 (c, d). 
(Рис. 2b,d). Третичные выделения $\gamma^{\prime}$-фазы размером менее 0.1 мкм в состояниях 2 и 3 иногда наблюдались по границам $\gamma$-зерен, их объемная доля не превышала $1 \%$. После первой стадии старения при температуре $860^{\circ} \mathrm{C}$ во всех исследованных состояниях наблюдалось выделение незначительной объемной доли дисперсных выделений карбидов вдоль межзеренных и межфазных границ (Рис. 1b,c, Рис. 2). Таким образом, обработка на твердый раствор при $1170^{\circ} \mathrm{C}$ (состояние 2) позволила сохранить относительно мелкий размер $\gamma$-зерен [7-9], при этом средний размер вторичной $\gamma^{\prime}$-фазы составил $d_{\gamma^{\prime}}=0.13 \pm 0.01$ мкм. Полученные микроструктурные параметры являются хорошей предпосылкой для достижения улучшенных механических свойств.

\section{2. Длительная прочность и сопротивление ползучести сплава}

Из Рис. 3 видно, что сопротивление ползучести значительно увеличивается при переходе от состояния 1 к состоянию 2 и 3 . В исследуемых состояниях сплава первая стадия ползучести была довольно слабовыраженной и только в состоянии 1 она сопровождалась некоторым снижением скорости ползучести. В состояниях 2 и 3 на первой стадии ползучести наблюдались незначительные осцилляции скорости ползучести (Рис. 3 b). Вторая стадия ползучести для всех исследуемых состояний сопровождалась некоторыми колебаниями скорости ползучести с незначительным ее увеличением (состояния 2 и 3) или примерно постоянной скоростью ползучести (состояние 1) (Рис. $3 \mathrm{~b})$. Колебания скорости ползучести ранее наблюдали [10] для деформированного никелевого сплава. Как известно, в микроструктуре деформированных сплавов всегда содержится повышенная плотность дислокаций, что может вызывать движение и образование новых дислокаций с периодическим торможением движения дислокаций, обусловленным взаимодействием с дисперсными выделениями, способствуя некоторой нестабильности скорости ползучести. Видно, что средняя скорость

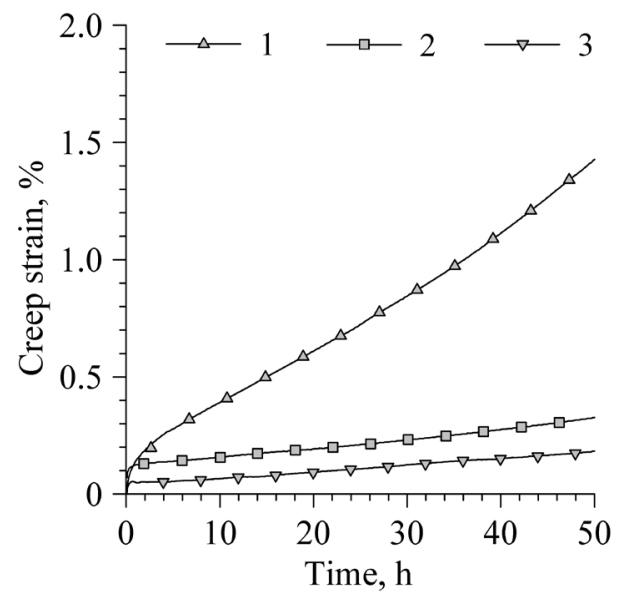

a ползучести значительно уменьшается при переходе от состояния 1 к состоянию 2, что, учитывая небольшое различие в размере $\gamma$-зерен в указанных состояниях, объясняется, прежде всего, увеличением объемной доли выделений вторичной $\gamma^{\prime}$-фазы. В состояниях 2 и 3 была обеспечена близкая между собой и значительно более низкая средняя скорость ползучести на второй стадии ползучести в сравнении с состоянием 1. Наименьшая скорость ползучести была получена для состояния 3.

У литых и монокристаллических жаропрочных никелевых сплавов первая стадия ползучести, как правило, сопровождается значительным снижением скорости ползучести $[12,15]$. Для состояний сплава $1-3$, полученных с помощью ДТО, этого почти не наблюдалось, что, по-видимому, связано с относительно легким зарождением и движением дислокаций в области межзеренных и межфазных границ благодаря относительно малому размеру $\gamma$-зерен. Между тем, образцы сплава во всех исследованных состояниях с большим запасом прочности не разрушились после 100-часовых испытаний при температуре $750^{\circ} \mathrm{C}$ и нагрузке $700 \mathrm{MПа.}$

Далее для состояния 2, благодаря меньшему размеру $\gamma$-зерен, были проведены испытания на ползучесть при температурах 750, 800 и $850^{\circ} \mathrm{C}$. При температурах испытания 750 и $800^{\circ} \mathrm{C}$ (Рис. 4) наблюдались слабовыраженные первая и вторая стадии ползучести с некоторым повышением скорости ползучести. При температуре испытания $850^{\circ} \mathrm{C}$ наблюдалась довольно протяженная первая стадия с уменьшением скорости ползучести, и короткой второй стадией, сопровождавшейся увеличением скорости ползучести (Рис. $4 \mathrm{~b}$ ). Из Рис. 4 а видно, что при $850^{\circ} \mathrm{C}$ вторая стадия довольно быстро сменяется третьей стадией, характеризующейся резким увеличением скорости ползучести. Для состояния 2 сплава были достигнуты следующие значения длительной прочности: при $650^{\circ} \mathrm{C} / 1200$ МПа - время до разрушения 201 ч, деформация до разрушения $5.4 \%$; при $750^{\circ} \mathrm{C} / 765 \mathrm{MПа} \mathrm{-}$ 124.6 ч и $5.0 \%$; при $800^{\circ} \mathrm{C} / 550 \mathrm{MПа}-78.9$ ч и $22.1 \%$; при $850^{\circ} \mathrm{C} / 400 \mathrm{MПа}-38.6$ ч и $18.4 \%$.

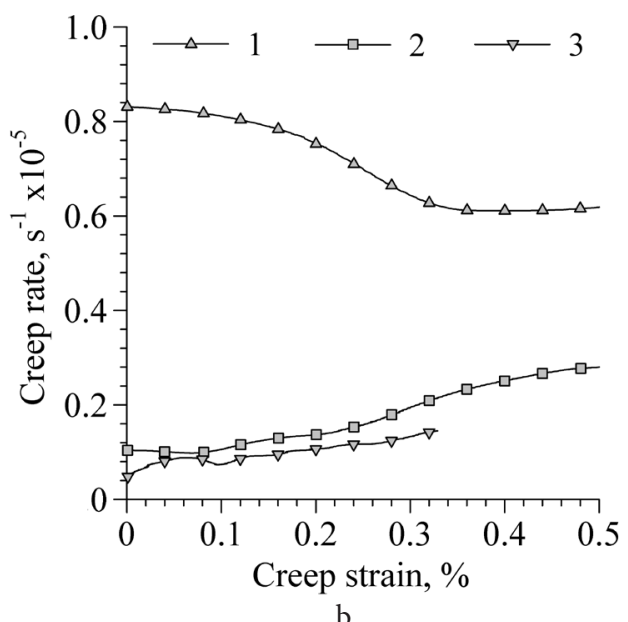

Рис. 3. Данные по ползучести сплава, полученные для состояний 1-3 при $750^{\circ} \mathrm{C} / 700$ МПа: кривые ползучести (а); зависимости скорости ползучести от деформации ползучести (b).

Fig. 3. Creep behavior of the superalloy in conditions $1-3$ at $750^{\circ} \mathrm{C} / 700 \mathrm{MPa}$ : the creep curves (a); the creep strain rate versus the strain value (b). 
На Рис. 5 представлены зависимости напряжения от параметра Ларсона - Миллера для исследуемого сплава и для сплавов ЭП741НП $[13,16]$ и Udimet 720 [13]. Видно, что сплав СДЖС-15 в состояниях 2 и 3 имеет более высокие значения длительной прочности. Состояния 2 и 3 показали близкие значения длительной прочности. Таким образом, оптимальной температурой обработки на твердый раствор, учитывая более высокие прочностные свойства сплава в состоянии 2, чем в состоянии 3 [17], является $T=1170^{\circ} \mathrm{C}$.

\section{3. Микроструктурные изменения после испьтания образияов на длительную прочность}

На Рис. 6 показано, что длительная деформация в режиме ползучести не привела к значительному изменению микроструктуры, топологически плотноупакованные фазы не были обнаружены. Деформация ползучести привела к зарождению трещин по межзеренным и межфазным границам. По-видимому, это связано

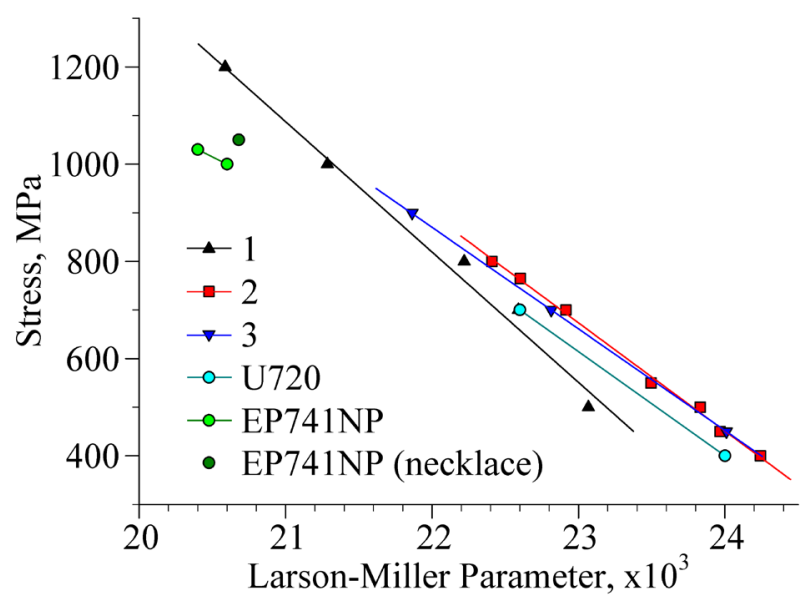

Puc. 5. (Color online) График зависимости напряжения от параметра Ларсона-Миллера для сплава СДЖС-15 в состоянии 1-3 и других ЖНС.

Fig. 5. (Color online) Larson-Miller parameter plotted for the superalloy SDZhS-15 in conditions 1-3 and the others superalloys.

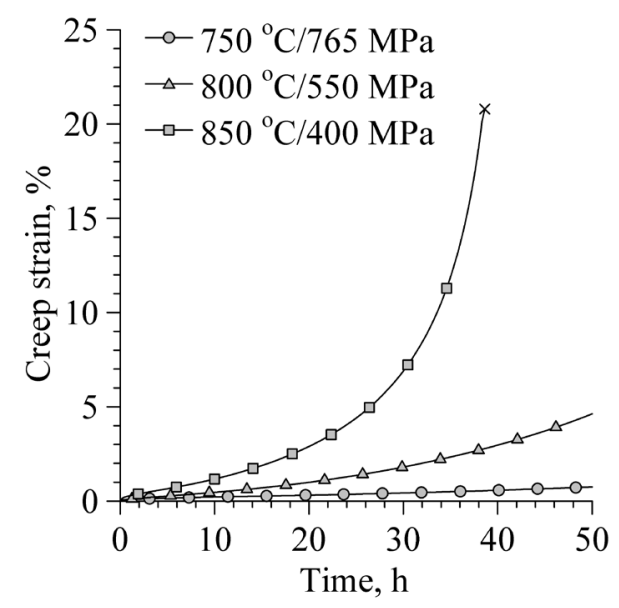

a

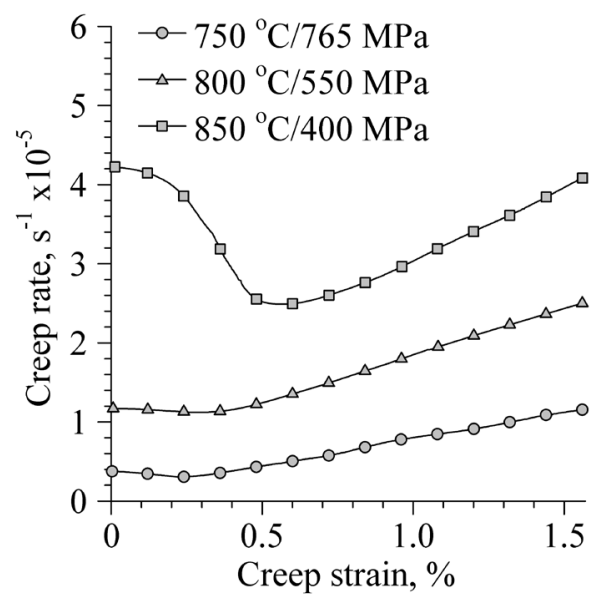

$\mathrm{b}$

Рис. 4. Ползучесть никелевого сплава в состоянии 2 при условиях испытания $750-850^{\circ} \mathrm{C} / 765-400$ МПа: кривые ползучести (a); зависимость скорости деформации ползучести от деформации (b).

Fig. 4. Creep behavior of the superalloy in condition 2 at $750-850^{\circ} \mathrm{C} / 765-400 \mathrm{MPa}$ : the creep curves (a); the creep strain rate versus the strain value (b).

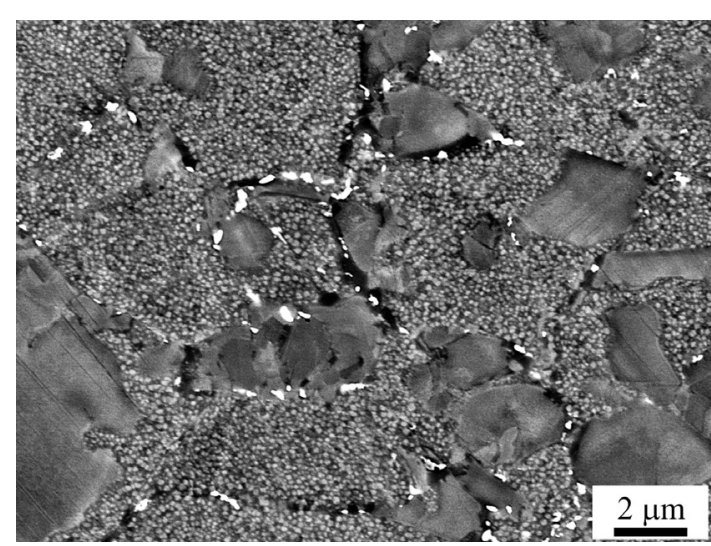

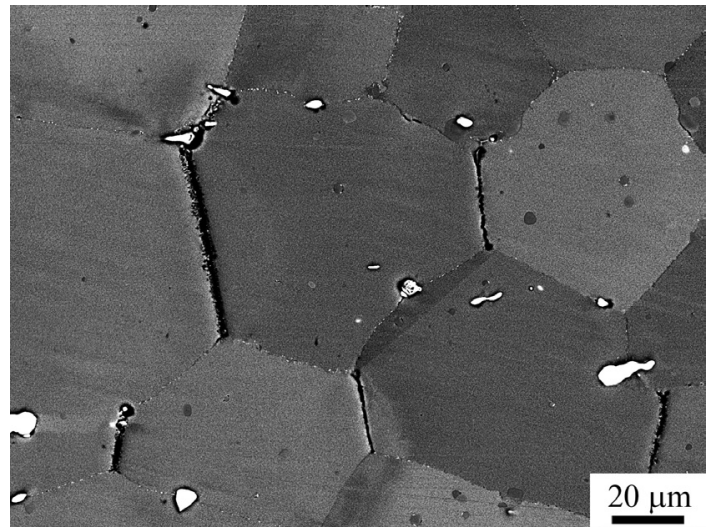

b

Рис. 6. Микроструктура сплава, полученная вблизи зоны разрушения образцов после испытаний на ползучесть при $850^{\circ} \mathrm{C} / 450 \mathrm{MПа:}$ состояние 2 (a), состояние 3 (b) (SEM, BSE). Ось нагружения - горизонтальная.

Fig. 6. The microstructural images obtained near the fracture zones of samples after creep tests at $850^{\circ} \mathrm{C} / 450 \mathrm{MPa}$ : condition 2 (a); condition 3 (b) (SEM, BSE). The loading axis was horizontal. 
с развитием диффузии и проскальзывания по границам в процессе испытания. Пограничная первичная $\gamma^{\prime}$-фаза и карбиды также могли способствовать зарождению пор с последующим формированием микротрещин (Рис. 6). В состоянии 2 в пределах частиц крупной первичной $\gamma^{\prime}$-фазы отмечались следы дислокационного скольжения (Рис.6а), которые не были обнаружены в исходных состояниях сплава (Рис. 1с и Рис. $2 \mathrm{~b}, \mathrm{~d}$ ). Развитие деформации в первичных выделениях $\gamma^{\prime}$-фазы, вероятно, способствовало колебаниям скорости ползучести в процессе испытаний.

\section{4. Заключение}

Обработка на твердый раствор деформированного сплава СДЖС-15 при температуре $1170^{\circ} \mathrm{C}$ является оптимальной, так как позволяет сохранить мелкозернистую структуру. Показано, что сплав СДЖС-15 в оптимальном состоянии имеет повышенную длительную прочность по сравнению со сплавами ЭП741НП и Udimet 720. Изучение микроструктуры образцов, испытанных на ползучесть, показало, что снижение сопротивления ползучести при $850^{\circ} \mathrm{C}$ может быть связано с повышенным коэффициентом диффузии вдоль межзеренных и межфазных границ, что приводит к образованию вдоль них пор и микротрещин.

Благодарности / Acknowledgements. Paбота выполнена в рамках Госзадания ИПСМ РАН (№АAАА-А17-117041310215-4). Экспериментальные исследования были выполнень на базе Центра коллективного пользования научным оборудованием ИПСМ РАН. / The present work was performed within the framework of the State Assignment of the Institute for Metals Superplasticity Problems of the Russian Academy of Sciences (No. AAAA-A17-117041310215-4). The work was performed using the facilities of the shared services center "Structural and Physical-Mechanical Studies of Materials" at the Institute for Metals Superplasticity Problems of Russian Academy of Sciences.

\section{Литература/References}

1. R.C. Reed. The superalloys: Fundamentals and Applications. Cambridge University Press (2006) 372 p. Crossref

2. Sh.Kh. Mukhtarov, V.M. Imayev, A. V. Logunov, Yu. N. Shmotin, A.M. Mikhailov, R.A. Gaisin,
R. V. Shakhov, A.A. Ganeev, R.M. Imayev. Mater. Sci. Technol. 35, 1605 (2019). Crossref

3. V.M. Imayev, S.K. Mukhtarov, A.V. Logunov, A.A. Ganeev, R. V. Shakhov, R.M. Imayev. Letters on Materials. 9 (2), 249 (2019). (in Russian) [B. М. Имаев, Ш.Х. Мухтаров, А.В. Логунов, А.А. Ганеев, Р.В. Шахов, Р. М. Имаев. Письма о материалах. 9 (2), 249 (2019).] Crossref

4. L. Thébaud, P. Villechaise, J. Cormier, C. Crozet, A. Devaux, D. Béchet, J.-M. Franchet, A. Organista, F. Hamon. Metals. 5, 2236 (2015). Crossref

5. C. Xu, F. Liu, L. Huang, L. Jiang. Metals. 8, 4 (2018). Crossref

6. N. Mrozowski, G. Hénaff, F. Hamon, A.-L. Rouffié, J.-M. Franchet, J. Cormier, P. Villechaise. Metals. 10, 426 (2020). Crossref

7. E. V. Filonova, M.M. Bakradze, A. Ya. Kochubei, N.L. Babelin. Aviation materials and technologies. 3, 10 (2014). (in Russian) [Е. В. Филонова, М. М. Бакрадзе, А.Я. Кочубей, Н.Л. Вавилин. Авиационные материалы и технологии. 3, 10 (2014).] Crossref

8. Patent RF №2653386 C1, 05.08.2018. (in Russian) [Патент РФ № 2653386 C1, 05.08.2018.]

9. B. S. Lomberg, S. V. Hovsepyan, M. M. Bakradze. Aviation materials and technologies. 2, 3 (2010). (in Russian) [Б.С. Ломберг, С.В. Овсепян, М.М. Бакрадзе. Авиационные материалы и технологии. 2, 3 (2010).]

10. T. Tian, C. Ge, X. Li, Z. Hao, S. Peng, C. Jia. Metals. 10, 454 (2020). Crossref

11. F. R. Larson, J. Miller. Trans. ASME. 74, 765 (1952).

12. L.S. Mataveli, J. Cormier, P. Villechaise, D. Bertheau, G. Benoit, G. Cailletaud, L. Marcin. Mater. High Temp. 33, 361 (2016). Crossref

13. J. Radavich, D. Furrer. Superalloys 2004 (ed. by K.A. Green). TMS, Warrendale, PA, USA (2004) pp. 381-390. Crossref

14. Y.L. Hu, Y.L. Li, S.Y. Zhang, X. Lin, Z.H. Wang, W.D. Huang. Mater. Sci.Eng. A. 772, 138711 (2020). Crossref

15. D. Bürger, A.B. Parsa, M. Ramsperger, C. Körner, G. Eggeler. Mater. Sci.Eng. A. 762, 138098 (2019). Crossref

16. A. A. Ganeev, V.A. Valitov, F.Z. Utyashev, V.M. Imaev. Physics of Metals and Metallography. 120 (4), 410 (2019). Crossref

17. K.S. Mukhtarova, R.V. Shakhov, A.A. Ganeev, S. K. Mukhtarov, A. V. Logunov, V. M. Imayev. IOP Conf. Series: Mater. Sci. Eng. 1008, 012010 (2020). $\underline{\text { Crossref }}$ 